\title{
Approximation of the Euclidean ball by polytopes
}

\author{
by \\ Monika Ludwig (Wien), Carsten Schütt (Kiel) \\ and Elisabeth Werner (Cleveland, OH, and Lille)
}

Abstract. There is a constant $c$ such that for every $n \in \mathbb{N}$, there is an $N_{n}$ so that for every $N \geq N_{n}$ there is a polytope $P$ in $\mathbb{R}^{n}$ with $N$ vertices and

$$
\operatorname{vol}_{n}\left(B_{2}^{n} \triangle P\right) \leq c \operatorname{vol}_{n}\left(B_{2}^{n}\right) N^{-\frac{2}{n-1}}
$$

where $B_{2}^{n}$ denotes the Euclidean unit ball of dimension $n$.

1. Main results. Let $C$ and $K$ be two convex bodies in $\mathbb{R}^{n}$. The Euclidean ball with center 0 and radius $r$ is denoted by $B_{2}^{n}(r)$. The ball $B_{2}^{n}(1)$ is denoted by $B_{2}^{n}$. Let $K$ be a convex body in $\mathbb{R}^{n}$ with $C^{2}$-boundary $\partial K$ and everywhere strictly positive curvature $\kappa$. Then

$$
\begin{aligned}
\lim _{N \rightarrow \infty} \frac{\inf \left\{\operatorname{vol}_{n}(K \backslash P) \mid P \subseteq K \text { and } P \text { has at most } N \text { vertices }\right\}}{} & N^{-\frac{2}{n-1}} \\
& =\frac{1}{2} \operatorname{del}_{n-1}\left(\int_{\partial K} \kappa(x)^{\frac{1}{n+1}} d \mu_{\partial K}(x)\right)^{\frac{n+1}{n-1}}
\end{aligned}
$$

where $\mu_{\partial K}$ denotes the surface measure of $\partial K$. This theorem gives asymptotically the order of best approximation of a convex body $K$ by polytopes contained in $K$ with a fixed number of vertices. It was proved by McClure and Vitale $[\mathrm{McV}]$ in dimension 2 and by Gruber [Gr2] for general $n$. The constant del $_{n-1}$ is positive and depends on the dimension $n$ only. Its order of magnitude can be computed by considering the case $K=B_{2}^{n}$. This has been done in [GRS1] and [GRS2] by Gordon, Reisner and Schütt, namely there are numerical constants $a$ and $b$ such that

$$
\text { an } \leq \operatorname{del}_{n-1} \leq \text { bn } .
$$

2000 Mathematics Subject Classification: Primary 52A20.

Key words and phrases: convex body, approximation by polytopes.

Research of E. Werner partially supported by a grant from the National Science Foundation, from a NATO Collaborative Linkage Grant, and from an NSF Advance Opportunity Grant. Research of all authors was supported in part by the European Network PhD, MCRN-511953. 
The constant del $\mathrm{l}_{n-1}$ was determined more precisely by Mankiewicz and Schütt [MaS1], [MaS2]. They showed that

$$
\begin{aligned}
& \frac{n-1}{n+1}\left(\operatorname{vol}_{n-1}\left(B_{2}^{n-1}\right)\right)^{-\frac{2}{n-1}} \\
& \quad \leq \operatorname{del}_{n-1} \leq\left(1+\frac{c \ln n}{n}\right) \frac{n-1}{n+1}\left(\operatorname{vol}_{n-1}\left(B_{2}^{n-1}\right)\right)^{-\frac{2}{n-1}}
\end{aligned}
$$

where $c$ is a numerical constant. In particular,

$$
\lim _{n \rightarrow \infty} \frac{\operatorname{del}_{n-1}}{n}=\frac{1}{2 \pi e} .
$$

What happens if we drop the condition that the polytopes have to be contained in the convex body and allow all polytopes have at most $N$ vertices? How much better can we approximate the Euclidean ball?

In [Lud] it was shown that for all convex bodies $K$ whose boundary is twice continuously differentiable and whose curvature is everywhere strictly positive,

$$
\begin{aligned}
\lim _{N \rightarrow \infty} \frac{\inf \left\{\operatorname{vol}_{n}(K \triangle P) \mid P \text { is a polytope with at most } N \text { vertices }\right\}}{N^{-\frac{2}{n-1}}} \\
=\frac{1}{2} \operatorname{ldel}_{n-1}\left(\int_{\partial K} \kappa(x)^{\frac{1}{n+1}} d \mu_{\partial K}(x)\right)^{\frac{n+1}{n-1}} .
\end{aligned}
$$

The constant $\operatorname{ldel}_{n-1}$ is positive and depends only on $n$. Clearly, from the above mentioned results it follows that $\operatorname{ldel}_{n-1} \leq \mathrm{cn}$. On the other hand, it has been shown in [Bö] that for a polytope $P$ with at most $N$ vertices,

$$
\operatorname{vol}_{n}\left(B_{2}^{n} \triangle P\right) \geq \frac{1}{67 e^{2} \pi} \frac{1}{n} \operatorname{vol}_{n}\left(B_{2}^{n}\right) N^{-\frac{2}{n-1}} .
$$

Thus between the upper and lower estimate for $\operatorname{ldel}_{n-1}$ there is a gap of order $n^{2}$. In this paper we narrow this gap by showing that $\operatorname{ldel}_{n-1} \leq c$ where $c$ is a numerical constant.

THEOREM 1. There is a constant $c$ such that for every $n \in \mathbb{N}$ there is an $N_{n}$ so that for every $N \geq N_{n}$ there is a polytope $P$ in $\mathbb{R}^{n}$ with $N$ vertices such that

$$
\operatorname{vol}_{n}\left(B_{2}^{n} \triangle P\right) \leq c \operatorname{vol}_{n}\left(B_{2}^{n}\right) N^{-\frac{2}{n-1}} .
$$

Gruber [Gr2] also showed

$$
\begin{array}{r}
\lim _{N \rightarrow \infty} \frac{\inf \left\{\operatorname{vol}_{n}(K \triangle P) \mid K \subseteq P \text { and } P \text { is a polytope with at most } N \text { facets }\right\}}{N^{-\frac{2}{n-1}}} \\
=\frac{1}{2} \operatorname{div}_{n-1}\left(\int_{\partial K} \kappa(x)^{\frac{1}{n+1}} d \mu_{\partial K}(x)\right)^{\frac{n+1}{n-1}}
\end{array}
$$


where $\operatorname{div}_{n-1}$ is a positive constant that depends on $n$ only. It is easy to show [Lud, MaS1] that there are numerical constants $a$ and $b$ such that an $\leq \operatorname{div}_{n-1} \leq b n$.

Ludwig [Lud] showed that for general polytopes

$$
\begin{aligned}
\lim _{N \rightarrow \infty} \frac{\inf \left\{\operatorname{vol}_{n}(K \triangle P) \mid P \text { is a polytope with at most } N \text { facets }\right\}}{N^{-\frac{2}{n-1}}} \\
=\frac{1}{2} \operatorname{ldiv}_{n-1}\left(\int_{\partial K} \kappa(x)^{\frac{1}{n+1}} d \mu_{\partial K}(x)\right)^{\frac{n+1}{n-1}}
\end{aligned}
$$

where $\operatorname{ldiv}_{n-1}$ is a positive constant that depends on $n$ only. Clearly, $\operatorname{ldiv}_{n-1}$ $\leq c n$ and Böröczky [Bö] showed that for polytopes $P$ with $N$ facets,

$$
\operatorname{vol}_{n}\left(B_{2}^{n} \triangle P\right) \geq \frac{1}{67 e^{2} \pi} \frac{1}{n} \operatorname{vol}_{n}\left(B_{2}^{n}\right) N^{-\frac{2}{n-1}} .
$$

Thus again, there is a gap between the upper and lower estimates for $\operatorname{ldiv}_{n-1}$ of order $n^{2}$. We narrow this gap by a factor of $n$.

THEOREM 2. There is a constant $c$ such that for every $n \in \mathbb{N}$ and for every $M \geq 10^{(n-1) / 2}$ and all polytopes $P$ in $\mathbb{R}^{n}$ with $M$ facets we have

$$
\operatorname{vol}_{n}\left(B_{2}^{n} \triangle P\right) \geq c \operatorname{vol}_{n}\left(B_{2}^{n}\right) M^{-\frac{2}{n-1}} .
$$

For additional information on asymptotic approximation see [Gr1], [Gr3], [Sch].

2. Proof of Theorem 1. We need the following lemmas.

Lemma 3 (Stirling's formula). For all $x>0$,

$$
\sqrt{2 \pi x} x^{x} e^{-x}<\Gamma(x+1)<\sqrt{2 \pi x} x^{x} e^{-x} e^{1 / 12 x} .
$$

The following lemma is due to J. Müller [Mü].

Lemma 4 ([Mü] $)$. Let $\mathbb{E}\left(\partial B_{2}^{n}, N\right)$ be the expected volume of a random polytope of $N$ points that are independently chosen on the boundary of the Euclidean ball $B_{2}^{n}$ with respect to the normalized surface measure. Then

$$
\begin{aligned}
& \lim _{N \rightarrow \infty} \frac{\operatorname{vol}_{n}\left(B_{2}^{n}\right)-\mathbb{E}\left(\partial B_{2}^{n}, N\right)}{N^{-\frac{2}{n-1}}} \\
& \quad=\frac{(n-1)^{\frac{n+1}{n-1}}\left(\operatorname{vol}_{n-1}\left(\partial B_{2}^{n}\right)\right)^{\frac{n+1}{n-1}}}{\left(\operatorname{vol}_{n-2}\left(\partial B_{2}^{n-1}\right)\right)^{\frac{2}{n-1}}} \frac{\Gamma\left(n+1+\frac{2}{n-1}\right)}{2(n+1) !} .
\end{aligned}
$$

The following lemma can be found in [Mil], [SW, p. 317], and [Zä]. Let $\left[x_{n}, \ldots, x_{n}\right]$ be the convex hull of $x_{1}, \ldots, x_{n}$. 
LEMMA 5 ([Mil]).

$$
\begin{aligned}
& d \mu_{\partial B_{2}^{n}}\left(x_{1}\right) \cdots d \mu_{\partial B_{2}^{n}}\left(x_{n}\right) \\
= & (n-1) ! \frac{\operatorname{vol}_{n-1}\left(\left[x_{1}, \ldots, x_{n}\right]\right)}{\left(1-p^{2}\right)^{n / 2}} d \mu_{\partial B_{2}^{n} \cap H}\left(x_{1}\right) \cdots d \mu_{\partial B_{2}^{n} \cap H}\left(x_{n}\right) d p d \mu_{\partial B_{2}^{n}}(\xi)
\end{aligned}
$$

where $\xi$ is the normal to the plane $H$ through $x_{1}, \ldots, x_{n}$ and $p$ is the distance of the plane $H$ to the origin.

LEMMA 6 ([Mil]).

$$
\begin{array}{r}
\int_{\partial B_{2}^{n}(r)} \cdots \int_{\partial B_{2}^{n}(r)}\left(\operatorname{vol}_{n}\left(\left[x_{1}, \ldots, x_{n+1}\right]\right)\right)^{2} d \mu_{\partial B_{2}^{n}(r)}\left(x_{1}\right) \cdots d \mu_{\partial B_{2}^{n}(r)}\left(x_{n+1}\right) \\
=\frac{(n+1) r^{n^{2}+2 n-1}}{n ! n^{n}}\left(\operatorname{vol}_{n-1}\left(\partial B_{2}^{n}\right)\right)^{n+1} .
\end{array}
$$

For a given hyperplane $H$ that does not contain the origin we denote by $H^{+}$the halfspace containing the origin and by $H^{-}$the halfspace not containing the origin. A cap $C$ of the Euclidean ball $B_{2}^{n}$ is the intersection of a halfspace $H^{-}$with $B_{2}^{n}$. The radius of such a cap is the radius of the $(n-1)$-dimensional ball $B_{2}^{n} \cap H$.

LEMMA 7 ([SW]). Let $H$ be a hyperplane, $p$ its distance from the origin and $s$ the normalized surface area of $\partial B_{2}^{n} \cap H^{-}$, i.e.

$$
s=\frac{\operatorname{vol}_{n-1}\left(\partial B_{2}^{n} \cap H^{-}\right)}{\operatorname{vol}_{n-1}\left(\partial B_{2}^{n}\right)} .
$$

Then

$$
\frac{d p}{d s}=-\frac{1}{\left(1-p^{2}\right)^{\frac{n-3}{2}}} \frac{\operatorname{vol}_{n-1}\left(\partial B_{2}^{n}\right)}{\operatorname{vol}_{n-2}\left(\partial B_{2}^{n-1}\right)} .
$$

LEMma 8 ([SW, Lemma 3.13]). Let $C$ be a cap of a Euclidean ball with radius 1 . Let $u$ be the surface area of this cap and $r$ its radius. Then

$$
\begin{aligned}
& \left(\frac{u}{\operatorname{vol}_{n-1}\left(B_{2}^{n-1}\right)}\right)^{\frac{1}{n-1}}-\frac{1}{2(n+1)}\left(\frac{u}{\operatorname{vol}_{n-1}\left(B_{2}^{n-1}\right)}\right)^{\frac{3}{n-1}} \\
& \quad-c\left(\frac{u}{\operatorname{vol}_{n-1}\left(B_{2}^{n-1}\right)}\right)^{\frac{5}{n-1}} \leq r(u) \leq\left(\frac{u}{\operatorname{vol}_{n-1}\left(B_{2}^{n-1}\right)}\right)^{\frac{1}{n-1}}
\end{aligned}
$$

where $c$ is a numerical constant.

The right hand inequality is immediate, since $u \geq r^{n-1} \operatorname{vol}_{n-1}\left(B_{2}^{n-1}\right)$.

Proof of Theorem 1. The approximating polytope is obtained in a probabilistic way. We consider a Euclidean ball that is slightly bigger than the ball with radius 1 , by a carefully chosen factor. We choose $N$ points randomly in the bigger ball and we take their convex hull. With large probability there is a random polytope that fits our requirements. 
For technical reasons we choose random points in a Euclidean ball of radius 1 and we approximate a slightly smaller Euclidean ball, say with radius $1-c$ where $c=c_{n, N}$ depends on $n$ and $N$ only.

We now compute the expected volume difference between $B_{2}^{n}(1-c)$ and a random polytope $\left[x_{1}, \ldots, x_{N}\right]$ whose vertices are chosen randomly from the boundary of $B_{2}^{n}$. Note that random polytopes are simplicial with probability 1 . We want to estimate the expected volume difference

$$
\begin{aligned}
& \mathbb{E} \operatorname{vol}_{n}\left(B_{2}^{n}(1-c) \triangle P_{N}\right) \\
& \quad=\int_{\partial B_{2}^{n}} \cdots \int_{\partial B_{2}^{n}} \operatorname{vol}_{n}\left(B_{2}^{n}(1-c) \triangle\left[x_{1}, \ldots, x_{N}\right]\right) d \mathbb{P}\left(x_{1}\right) \cdots d \mathbb{P}\left(x_{N}\right)
\end{aligned}
$$

where $\mathbb{P}$ denotes the uniform probability measure on $\partial B_{2}^{n}$. Since the volume difference between $B_{2}^{n}(1-c)$ and a polytope $P_{N}=\left[x_{1}, \ldots, x_{N}\right]$ is

$$
\begin{aligned}
& \operatorname{vol}_{n}\left(B_{2}^{n}(1-c) \triangle P_{N}\right) \\
& \quad=\operatorname{vol}_{n}\left(B_{2}^{n} \backslash B_{2}^{n}(1-c)\right)-\operatorname{vol}_{n}\left(B_{2}^{n} \backslash P_{N}\right)+2 \operatorname{vol}_{n}\left(B_{2}^{n}(1-c) \cap P_{N}^{\mathrm{c}}\right),
\end{aligned}
$$

the above expression equals

$$
\begin{aligned}
\operatorname{vol}_{n}\left(B_{2}^{n} \backslash B_{2}^{n}(1-c)\right) & \\
& -\int_{\partial B_{2}^{n}} \cdots \int_{\partial B_{2}^{n}} \operatorname{vol}_{n}\left(B_{2}^{n} \backslash\left[x_{1}, \ldots, x_{N}\right]\right) d \mathbb{P}\left(x_{1}\right) \cdots d \mathbb{P}\left(x_{N}\right) \\
& +2 \int_{\partial B_{2}^{n}} \cdots \int_{\partial B_{2}^{n}} \operatorname{vol}_{n}\left(B_{2}^{n}(1-c) \cap\left[x_{1}, \ldots, x_{N}\right]^{\mathrm{c}}\right) d \mathbb{P}\left(x_{1}\right) \cdots d \mathbb{P}\left(x_{N}\right) .
\end{aligned}
$$

For given $N$ we choose $c$ such that

$$
\begin{aligned}
\operatorname{vol}_{n}\left(B_{2}^{n} \backslash\right. & \left.\backslash B_{2}^{n}(1-c)\right) \\
& =\int_{\partial B_{2}^{n}} \cdots \int_{\partial B_{2}^{n}} \operatorname{vol}_{n}\left(B_{2}^{n} \backslash\left[x_{1}, \ldots, x_{N}\right]\right) d \mathbb{P}\left(x_{1}\right) \cdots d \mathbb{P}\left(x_{N}\right) .
\end{aligned}
$$

For this particular $c$ we have

$$
\begin{aligned}
& \int_{\partial B_{2}^{n}} \cdots \int_{\partial B_{2}^{n}} \operatorname{vol}_{n}\left(B_{2}^{n}(1-c) \triangle\left[x_{1}, \ldots, x_{N}\right]\right) d \mathbb{P}\left(x_{1}\right) \cdots d \mathbb{P}\left(x_{N}\right) \\
& \quad=2 \int_{\partial B_{2}^{n}} \cdots \int_{\partial B_{2}^{n}} \operatorname{vol}_{n}\left(B_{2}^{n}(1-c) \cap\left[x_{1}, \ldots, x_{N}\right]^{\mathrm{c}}\right) d \mathbb{P}\left(x_{1}\right) \cdots d \mathbb{P}\left(x_{N}\right) .
\end{aligned}
$$

By Lemma 4 the quantity $c$ is for large $N$ asymptotically equal to

$$
N^{-\frac{2}{n-1}}(n-1)^{\frac{n+1}{n-1}}\left(\frac{\operatorname{vol}_{n-1}\left(\partial B_{2}^{n}\right)}{\operatorname{vol}_{n-2}\left(\partial B_{2}^{n-1}\right)}\right)^{\frac{2}{n-1}} \frac{\Gamma\left(n+1+\frac{2}{n-1}\right)}{2(n+1) !} .
$$


In particular, for large enough $N$,

$$
\begin{aligned}
c \leq & \left(1+\frac{1}{n^{2}}\right) N^{-\frac{2}{n-1}}(n-1)^{\frac{n+1}{n-1}} \\
& \times\left(\frac{\operatorname{vol}_{n-1}\left(\partial B_{2}^{n}\right)}{\operatorname{vol}_{n-2}\left(\partial B_{2}^{n-1}\right)}\right)^{\frac{2}{n-1}} \frac{\Gamma\left(n+1+\frac{2}{n-1}\right)}{2(n+1) !}
\end{aligned}
$$

and

$$
\begin{aligned}
\left(1-\frac{1}{n^{2}}\right) N^{-\frac{2}{n-1}}(n & -1)^{\frac{n+1}{n-1}} \\
& \times\left(\frac{\operatorname{vol}_{n-1}\left(\partial B_{2}^{n}\right)}{\operatorname{vol}_{n-2}\left(\partial B_{2}^{n-1}\right)}\right)^{\frac{2}{n-1}} \frac{\Gamma\left(n+1+\frac{2}{n-1}\right)}{2(n+1) !} \leq c .
\end{aligned}
$$

Thus there are constants $a$ and $b$ such that

$$
a N^{-\frac{2}{n-1}} \leq c \leq b N^{-\frac{2}{n-1}} .
$$

We continue the computation of the expected volume difference:

$$
\begin{aligned}
& \int_{\partial B_{2}^{n}} \cdots \int_{\partial B_{2}^{n}} \operatorname{vol}_{n}\left(B_{2}^{n}(1-c) \triangle\left[x_{1}, \ldots, x_{N}\right]\right) d \mathbb{P}\left(x_{1}\right) \cdots d \mathbb{P}\left(x_{N}\right) \\
&=2 \int_{\partial B_{2}^{n}} \cdots \int_{\partial B_{2}^{n}} \operatorname{vol}_{n}\left(B_{2}^{n}(1-c) \cap\left[x_{1}, \ldots, x_{N}\right]^{\mathrm{c}}\right) \\
& \times \chi_{\left\{0 \in\left[x_{1}, \ldots, x_{N}\right]^{\circ}\right\}} d \mathbb{P}\left(x_{1}\right) \cdots d \mathbb{P}\left(x_{N}\right) \\
&+2 \int_{\partial B_{2}^{n}} \cdots \int_{\partial B_{2}^{n}} \operatorname{vol}_{n}\left(B_{2}^{n}(1-c) \cap\left[x_{1}, \ldots, x_{N}\right]^{\mathrm{c}}\right) \\
& \times \chi_{\left\{0 \notin\left[x_{1}, \ldots, x_{N}\right]^{\circ}\right\}} d \mathbb{P}\left(x_{1}\right) \cdots d \mathbb{P}\left(x_{N}\right) \\
& \leq 2 \int_{\partial B_{2}^{n}} \cdots \int_{\partial B_{2}^{n}} \operatorname{vol}_{n}\left(B_{2}^{n}(1-c) \cap\left[x_{1}, \ldots, x_{N}\right]^{\mathrm{c}}\right) \\
& \times \chi_{\left\{0 \in\left[x_{1}, \ldots, x_{N}\right]^{\circ}\right\}} d \mathbb{P}\left(x_{1}\right) \cdots d \mathbb{P}\left(x_{N}\right) \\
&+\operatorname{vol}_{n}\left(B_{2}^{n}\right) \int_{\partial B_{2}^{n}} \cdots \int_{\partial B_{2}^{n}} \chi_{\left\{0 \notin\left[x_{1}, \ldots, x_{N}\right]^{\circ}\right\}} d \mathbb{P}\left(x_{1}\right) \cdots d \mathbb{P}\left(x_{N}\right) .
\end{aligned}
$$

By a result of [Wen] the second summand equals

$$
\operatorname{vol}_{n}\left(B_{2}^{n}\right) 2^{-N+1} \sum_{k=0}^{n-1}\left(\begin{array}{c}
N-1 \\
k
\end{array}\right) \leq \operatorname{vol}_{n}\left(B_{2}^{n}\right) 2^{-N+1} n N^{n},
$$

so it is of much smaller order (essentially $2^{-N}$ ) than the first summand, which, as we shall see, is of the order of $N^{-2 /(n-1)}$. Therefore, in what follows we consider the first summand.

We introduce $\Phi_{j_{1}, \ldots, j_{n}}: \partial B_{2}^{n} \times \cdots \times \partial B_{2}^{n} \rightarrow \mathbb{R}$ where

$$
\Phi_{j_{1}, \ldots, j_{n}}\left(x_{1}, \ldots, x_{N}\right)=0
$$


if $\left[x_{j_{1}}, \ldots, x_{j_{n}}\right]$ is not an $(n-1)$-dimensional face of $\left[x_{1}, \ldots, x_{N}\right]$ or if 0 is not in $\left[x_{1}, \ldots, x_{N}\right]$, and

$\Phi_{j_{1}, \ldots, j_{n}}\left(x_{1}, \ldots, x_{N}\right)$

$$
=\operatorname{vol}_{n}\left(B_{2}^{n}(1-c) \cap\left[x_{1}, \ldots, x_{N}\right]^{\mathrm{c}} \cap \operatorname{cone}\left(x_{j_{1}}, \ldots, x_{j_{n}}\right)\right) \chi_{\left\{0 \in\left[x_{1}, \ldots, x_{N}\right]^{\circ}\right\}}
$$

if $\left[x_{j_{1}}, \ldots, x_{j_{n}}\right]$ is a facet of $\left[x_{1}, \ldots, x_{N}\right]$ and if $0 \in\left[x_{1}, \ldots, x_{N}\right]$. Here

$$
\operatorname{cone}\left(x_{1}, \ldots, x_{n}\right)=\left\{\sum_{i=1}^{n} a_{i} x_{i} \mid \forall i: a_{i} \geq 0\right\} \text {. }
$$

For all random polytopes $\left[x_{1}, \ldots, x_{N}\right]$ that contain 0 as an interior point,

$$
\mathbb{R}^{n}=\bigcup_{\left[x_{j_{1}}, \ldots, x_{j_{n}}\right] \text { is a facet of }\left[x_{1}, \ldots, x_{n}\right]} \operatorname{cone}\left(x_{j_{1}}, \ldots, x_{j_{n}}\right) .
$$

Then

$$
\begin{gathered}
\int_{\partial B_{2}^{n}} \cdots \int_{\partial B_{2}^{n}} \operatorname{vol}_{n}\left(B_{2}^{n}(1-c) \cap\left[x_{1}, \ldots, x_{N}\right]^{\mathrm{c}}\right) \chi_{\left\{0 \in\left[x_{1}, \ldots, x_{N}\right]^{\circ}\right\}} d \mathbb{P}\left(x_{1}\right) \cdots d \mathbb{P}\left(x_{N}\right) \\
\quad=\int_{\partial B_{2}^{n}} \cdots \int_{\partial B_{2}^{n}\left\{j_{1}, \ldots, j_{n}\right\} \subseteq\{1, \ldots, N\}} \sum_{j_{1}, \ldots, j_{n}}\left(x_{1}, \ldots, x_{N}\right) d \mathbb{P}\left(x_{1}\right) \cdots d \mathbb{P}\left(x_{N}\right)
\end{gathered}
$$

where we sum over all different subsets $\left\{j_{1}, \ldots, j_{n}\right\}$. The latter expression equals

$$
\left(\begin{array}{c}
N \\
n
\end{array}\right) \int_{\partial B_{2}^{n}} \cdots \int_{\partial B_{2}^{n}} \Phi_{1, \ldots, n}\left(x_{1}, \ldots, x_{N}\right) d \mathbb{P}\left(x_{1}\right) \cdots d \mathbb{P}\left(x_{N}\right) .
$$

Let $H$ be the hyperplane containing the points $x_{1}, \ldots, x_{n}$. The set of points where $H$ is not well defined has measure 0 and $\mathbb{P}^{N-n}\left(\left\{\left(x_{n+1}, \ldots, x_{N}\right) \mid\left[x_{1}, \ldots, x_{n}\right]\right.\right.$ is a facet of $\left[x_{1}, \ldots, x_{N}\right]$

$$
\text { and } \left.\left.0 \in\left[x_{1}, \ldots, x_{N}\right]\right\}\right)=\left(\frac{\operatorname{vol}_{n-1}\left(\partial B_{2}^{n} \cap H^{+}\right)}{\operatorname{vol}_{n-1}\left(\partial B_{2}^{n}\right)}\right)^{N-n} \text {. }
$$

Therefore the above expression equals

$$
\begin{aligned}
& \left(\begin{array}{c}
N \\
n
\end{array}\right) \int_{\partial B_{2}^{n}} \cdots \int_{\partial B_{2}^{n}}\left(\frac{\operatorname{vol}_{n-1}\left(\partial B_{2}^{n} \cap H^{+}\right)}{\operatorname{vol}_{n-1}\left(\partial B_{2}^{n}\right)}\right)^{N-n} \\
& \times \operatorname{vol}_{n}\left(B_{2}^{n}(1-c) \cap H^{-} \cap \operatorname{cone}\left(x_{1}, \ldots, x_{n}\right)\right) \chi_{\left\{0 \in\left[x_{1}, \ldots, x_{N}\right]^{\circ}\right\}} d \mathbb{P}\left(x_{1}\right) \cdots d \mathbb{P}\left(x_{n}\right) .
\end{aligned}
$$

Since $H^{-}$does not contain 0 this can be estimated by

$$
\begin{aligned}
& \left(\begin{array}{c}
N \\
n
\end{array}\right) \int_{\partial B_{2}^{n}} \cdots \int_{\partial B_{2}^{n}}\left(\frac{\operatorname{vol}_{n-1}\left(\partial B_{2}^{n} \cap H^{+}\right)}{\operatorname{vol}_{n-1}\left(\partial B_{2}^{n}\right)}\right)^{N-n} \\
& \quad \times \operatorname{vol}_{n}\left(B_{2}^{n}(1-c) \cap H^{-} \cap \operatorname{cone}\left(x_{1}, \ldots, x_{n}\right)\right) d \mathbb{P}\left(x_{1}\right) \cdots d \mathbb{P}\left(x_{n}\right) .
\end{aligned}
$$


By Lemma 5 the latter expression equals

$$
\begin{aligned}
\left(\begin{array}{c}
N \\
n
\end{array}\right) \frac{(n-1) !}{\left(\operatorname{vol}_{n-1}\left(\partial B_{2}^{n}\right)\right)^{n}} \int_{\partial B_{2}^{n}} \int_{0}^{1} \int_{\partial B_{2}^{n} \cap H} \cdots \int_{\partial B_{2}^{n} \cap H}\left(\frac{\operatorname{vol}_{n-1}\left(\partial B_{2}^{n} \cap H^{+}\right)}{\operatorname{vol}_{n-1}\left(\partial B_{2}^{n}\right)}\right)^{N-n} \\
\times \operatorname{vol}_{n}\left(B_{2}^{n}(1-c) \cap H^{-} \cap \operatorname{cone}\left(x_{1}, \ldots, x_{n}\right)\right) \\
\times \frac{\operatorname{vol}_{n-1}\left(\left[x_{1}, \ldots, x_{n}\right]\right)}{\left(1-p^{2}\right)^{n / 2}} d \mu_{\partial B_{2}^{n} \cap H}\left(x_{1}\right) \cdots d \mu_{\partial B_{2}^{n} \cap H}\left(x_{n}\right) d p d \mu_{\partial B_{2}^{n}}(\xi) .
\end{aligned}
$$

This in turn can be estimated by

$$
\begin{aligned}
& \left(\begin{array}{c}
N \\
n
\end{array}\right) \frac{(n-1) !}{\left(\operatorname{vol}_{n-1}\left(\partial B_{2}^{n}\right)\right)^{n}} \\
& \quad \times \int_{\partial B_{2}^{n}} \int_{1-1 / n}^{1} \int_{\partial B_{2}^{n} \cap H} \cdots \int_{\partial B_{2}^{n} \cap H}\left(\frac{\operatorname{vol}_{n-1}\left(\partial B_{2}^{n} \cap H^{+}\right)}{\operatorname{vol}_{n-1}\left(\partial B_{2}^{n}\right)}\right)^{N-n} \\
& \quad \times \operatorname{vol}_{n}\left(B_{2}^{n}(1-c) \cap H^{-} \cap \operatorname{cone}\left(x_{1}, \ldots, x_{n}\right)\right) \\
& \quad \times \frac{\operatorname{vol}_{n-1}\left(\left[x_{1}, \ldots, x_{n}\right]\right)}{\left(1-p^{2}\right)^{n / 2}} d \mu_{\partial B_{2}^{n} \cap H}\left(x_{1}\right) \cdots d \mu_{\partial B_{2}^{n} \cap H}\left(x_{n}\right) d p d \mu_{\partial B_{2}^{n}}(\xi)
\end{aligned}
$$

times a factor that is less than 2 provided that $N$ is sufficiently large. Indeed, for $p \leq 1-1 / n$,

$$
\begin{aligned}
\left(\frac{\operatorname{vol}_{n-1}\left(\partial B_{2}^{n} \cap H^{+}\right)}{\operatorname{vol}_{n-1}\left(\partial B_{2}^{n}\right)}\right)^{N-n} & \leq \exp \left(-(N-n) \frac{\operatorname{vol}_{n-1}\left(\partial B_{2}^{n} \cap H^{-}\right)}{\operatorname{vol}_{n-1}\left(\partial B_{2}^{n}\right)}\right) \\
& \leq \exp \left(-(N-n)\left(\frac{2}{n}-\frac{1}{n^{2}}\right)^{\frac{n-1}{2}} \frac{\operatorname{vol}_{n-1}\left(B_{2}^{n-1}\right)}{n \operatorname{vol}_{n}\left(B_{2}^{n}\right)}\right) \\
& \leq \exp \left(-\frac{N-n}{n^{(n+1) / 2}}\right)
\end{aligned}
$$

and the rest of the expression is bounded. We have

$$
\begin{aligned}
\operatorname{vol}_{n}\left(B_{2}^{n}(1-c) \cap H^{-}\right. & \left.\cap \operatorname{cone}\left(x_{1}, \ldots, x_{n}\right)\right) \\
\leq & \frac{p}{n} \max \left\{0,\left(\frac{1-c}{p}\right)^{n}-1\right\} \operatorname{vol}_{n-1}\left(\left[x_{1}, \ldots, x_{n}\right]\right) .
\end{aligned}
$$

This holds since $B_{2}^{n}(1-c) \cap H^{-} \cap \operatorname{cone}\left(x_{1}, \ldots, x_{n}\right)$ is contained in the cone cone $\left(x_{1}, \ldots, x_{n}\right)$, truncated between $H$ and the hyperplane parallel to $H$ at distance $1-c$ from 0 . Therefore, as $p \leq 1$ the above is at most 


$$
\begin{aligned}
\left(\begin{array}{c}
N \\
n
\end{array}\right) \frac{(n-1) !}{\left(\operatorname{vol}_{n-1}\left(\partial B_{2}^{n}\right)\right)^{n}} & \int_{\partial B_{2}^{n}} \int_{1-1 / n}^{1} \int_{\partial B_{2}^{n} \cap H} \cdots \int_{\partial B_{2}^{n} \cap H}\left(\frac{\operatorname{vol}_{n-1}\left(\partial B_{2}^{n} \cap H^{+}\right)}{\operatorname{vol}_{n-1}\left(\partial B_{2}^{n}\right)}\right)^{N-n} \\
& \times \frac{1}{n} \max \left\{0,\left(\frac{1-c}{p}\right)^{n}-1\right\} \frac{\left(\operatorname{vol}_{n-1}\left(\left[x_{1}, \ldots, x_{n}\right]\right)\right)^{2}}{\left(1-p^{2}\right)^{n / 2}} \\
& \times d \mu_{\partial B_{2}^{n} \cap H}\left(x_{1}\right) \cdots d \mu_{\partial B_{2}^{n} \cap H}\left(x_{n}\right) d p d \mu_{\partial B_{2}^{n}}(\xi) .
\end{aligned}
$$

By Lemma 6 this equals

$$
\begin{aligned}
\left(\begin{array}{c}
N \\
n
\end{array}\right) \frac{\left(\operatorname{vol}_{n-2}\left(\partial B_{2}^{n-1}\right)\right)^{n}}{\left(\operatorname{vol}_{n-1}\left(\partial B_{2}^{n}\right)\right)^{n}} & \frac{n}{(n-1)^{n-1}} \int_{\partial B_{2}^{n}} \int_{1-1 / n}^{1}\left(\frac{\operatorname{vol}_{n-1}\left(\partial B_{2}^{n} \cap H^{+}\right)}{\operatorname{vol}_{n-1}\left(\partial B_{2}^{n}\right)}\right)^{N-n} \\
& \times \frac{1}{n} \max \left\{0,\left(\frac{1-c}{p}\right)^{n}-1\right\} \frac{r^{n^{2}-2}}{\left(1-p^{2}\right)^{n / 2}} d p d \mu_{\partial B_{2}^{n}(\xi)}
\end{aligned}
$$

where $r$ denotes the radius of $B_{2}^{n} \cap H$. Since the integral does not depend on the direction $\xi$ and $r^{2}+p^{2}=1$ this is

$$
\begin{aligned}
& \left(\begin{array}{c}
N \\
n
\end{array}\right) \frac{\left(\operatorname{vol}_{n-2}\left(\partial B_{2}^{n-1}\right)\right)^{n}}{\left(\operatorname{vol}_{n-1}\left(\partial B_{2}^{n}\right)\right)^{n-1}} \frac{n}{(n-1)^{n-1}} \\
& \quad \times \int_{1-1 / n}^{1}\left(\frac{\operatorname{vol}_{n-1}\left(\partial B_{2}^{n} \cap H^{+}\right)}{\operatorname{vol}_{n-1}\left(\partial B_{2}^{n}\right)}\right)^{N-n} \frac{1}{n} \max \left\{0,\left(\frac{1-c}{p}\right)^{n}-1\right\} r^{n^{2}-n-2} d p .
\end{aligned}
$$

This equals

$$
\begin{aligned}
& \left(\begin{array}{c}
N \\
n
\end{array}\right) \frac{\left(\operatorname{vol}_{n-2}\left(\partial B_{2}^{n-1}\right)\right)^{n}}{\left(\operatorname{vol}_{n-1}\left(\partial B_{2}^{n}\right)\right)^{n-1}} \frac{n}{(n-1)^{n-1}} \\
& \quad \times \int_{1-1 / n}^{1-c}\left(\frac{\operatorname{vol}_{n-1}\left(\partial B_{2}^{n} \cap H^{+}\right)}{\operatorname{vol}_{n-1}\left(\partial B_{2}^{n}\right)}\right)^{N-n} \frac{1}{n}\left\{\left(\frac{1-c}{p}\right)^{n}-1\right\} r^{n^{2}-n-2} d p .
\end{aligned}
$$

Since $p \geq 1-1 / n$ and $c$ is of the order $N^{-2 /(n-1)}$, we have, for sufficiently large $N$,

$$
\frac{1}{n}\left\{\left(\frac{1-c}{p}\right)^{n}-1\right\} \leq 3(1-c-p) .
$$

Therefore, the previous expression can be estimated by an absolute constant times

$$
\begin{aligned}
\left(\begin{array}{c}
N \\
n
\end{array}\right) & \frac{\left(\operatorname{vol}_{n-2}\left(\partial B_{2}^{n-1}\right)\right)^{n}}{\left(\operatorname{vol}_{n-1}\left(\partial B_{2}^{n}\right)\right)^{n-1}} \frac{n}{(n-1)^{n-1}} \\
& \times \int_{1-1 / n}^{1-c}\left(\frac{\operatorname{vol}_{n-1}\left(\partial B_{2}^{n} \cap H^{+}\right)}{\operatorname{vol}_{n-1}\left(\partial B_{2}^{n}\right)}\right)^{N-n}(1-c-p) r^{n^{2}-n-2} d p .
\end{aligned}
$$


We choose

$$
s=\frac{\operatorname{vol}_{n-1}\left(\partial B_{2}^{n} \cap H^{-}\right)}{\operatorname{vol}_{n-1}\left(\partial B_{2}^{n}\right)}
$$

as our new variable under the integral. We apply Lemma 7 in order to change the variable under the integral

$$
\begin{aligned}
\left(\begin{array}{c}
N \\
n
\end{array}\right) \frac{\left(\operatorname{vol}_{n-2}\left(\partial B_{2}^{n-1}\right)\right)^{n-1}}{\left(\operatorname{vol}_{n-1}\left(\partial B_{2}^{n}\right)\right)^{n-2}} & \frac{n}{(n-1)^{n-1}} \\
& \times \int_{s(1-c)}^{1 / 2}(1-s)^{N-n}(1-c-p) r^{(n-1)^{2}} d s
\end{aligned}
$$

where the normalized surface area $s$ of the cap is a function of the distance $p$ of the hyperplane to 0 . Before we proceed we want to estimate $s(1-c)$. The radius $r$ and the distance $p$ satisfy $1=p^{2}+r^{2}$. We have

$$
r^{n-1} \frac{\operatorname{vol}_{n-1}\left(B_{2}^{n-1}\right)}{\operatorname{vol}_{n-1}\left(\partial B_{2}^{n}\right)} \leq s\left(\sqrt{1-r^{2}}\right) \leq \frac{1}{\sqrt{1-r^{2}}} r^{n-1} \frac{\operatorname{vol}_{n-1}\left(B_{2}^{n-1}\right)}{\operatorname{vol}_{n-1}\left(\partial B_{2}^{n}\right)} .
$$

To show this, we compare $s$ with the surface area of the intersection $B_{2}^{n} \cap H$ of the Euclidean ball and the hyperplane $H$. We have

$$
\frac{\operatorname{vol}_{n-1}\left(B_{2}^{n} \cap H\right)}{\operatorname{vol}_{n-1}\left(\partial B_{2}^{n}\right)}=r^{n-1} \frac{\operatorname{vol}_{n-1}\left(B_{2}^{n-1}\right)}{\operatorname{vol}_{n-1}\left(\partial B_{2}^{n}\right)} .
$$

Since the orthogonal projection onto $H$ maps $\partial B_{2}^{n} \cap H^{-}$onto $B_{2}^{n} \cap H$ the left hand inequality follows.

The right hand inequality follows again by considering the orthogonal projection onto $H$. The surface area of a surface element of $\partial B_{2}^{n} \cap H^{-}$equals the surface area of the one it is mapped to in $B_{2}^{n} \cap H$ divided by the cosine of the angle between the normal to $H$ and the normal to $\partial B_{2}^{n}$ at the given point. The cosine is always greater than $\sqrt{1-r^{2}}$.

For $p=1-c$ we have $r=\sqrt{2 c-c^{2}} \leq \sqrt{2 c}$. Therefore by (12) we get

$$
\begin{gathered}
s(1-c) \leq \frac{e^{1 / n}}{1-c} \frac{\operatorname{vol}_{n-1}\left(B_{2}^{n-1}\right)}{\operatorname{vol}_{n-1}\left(\partial B_{2}^{n}\right)} \\
\times\left\{2 N^{-\frac{2}{n-1}}(n-1)^{\frac{n+1}{n-1}}\left(\frac{\operatorname{vol}_{n-1}\left(\partial B_{2}^{n}\right)}{\operatorname{vol}_{n-2}\left(\partial B_{2}^{n-1}\right)}\right)^{\frac{2}{n-1}} \frac{\Gamma\left(n+1+\frac{2}{n-1}\right)}{2(n+1) !}\right\}^{\frac{n-1}{2}} \\
=\frac{e^{1 / n}}{1-c} \frac{1}{N}\left\{\frac{\Gamma\left(n+1+\frac{2}{n-1}\right)(n-1)}{(n+1) !}\right\}^{\frac{n-1}{2}} .
\end{gathered}
$$

The quantity $c$ is of the order $N^{-2 /(n-1)}$, therefore $1 /(1-c)$ is as close to 1 
as we desire for $N$ large enough. Moreover, for large $n$,

$$
\left(\frac{n-1}{n+1}\right)^{\frac{n-1}{2}}
$$

is asymptotically equal to $1 / e$. Therefore, for both $n$ and $N$ large enough,

$$
s(1-c) \leq e^{1 / 12} \frac{1}{e N}\left\{\frac{\Gamma\left(n+1+\frac{2}{n-1}\right)}{n !}\right\}^{\frac{n-1}{2}} .
$$

For $n$ sufficiently large,

$$
\left\{\frac{\Gamma\left(n+1+\frac{2}{n-1}\right)}{n !}\right\}^{\frac{n-1}{2}} \leq e^{1 / 12} n .
$$

Indeed, by Lemma 3,

$$
\frac{\Gamma\left(n+1+\frac{2}{n-1}\right)}{n !} \leq\left(1+\frac{2}{n(n-1)}\right)^{n+\frac{1}{2}}\left(n+\frac{2}{n-1}\right)^{\frac{2}{n-1}} e^{-\frac{2}{n-1}} e^{\frac{1}{12\left(n+\frac{2}{n-1}\right)}}
$$

and

$$
\begin{aligned}
& \left(\frac{\Gamma\left(n+1+\frac{2}{n-1}\right)}{n !}\right)^{\frac{n-1}{2}} \\
& \quad \leq \frac{1}{e}\left(1+\frac{2}{n(n-1)}\right)^{\frac{n-1}{2}\left(n+\frac{1}{2}\right)}\left(n+\frac{2}{n-1}\right) e^{\frac{n-1}{24\left(n+\frac{2}{n-1}\right)}} .
\end{aligned}
$$

The right hand expression is asymptotically equal to $n e^{1 / 24}$. Altogether,

$$
s(1-c) \leq e^{1 / 6} \frac{n}{e N} \text {. }
$$

Since $p=\sqrt{1-r^{2}}$ we get, for all $r$ with $0 \leq r \leq 1$,

$$
1-c-p=1-c-\sqrt{1-r^{2}} \leq \frac{1}{2} r^{2}+r^{4}-c .
$$

(The estimate is equivalent to $1-\frac{1}{2} r^{2}-r^{4} \leq \sqrt{1-r^{2}}$. The left hand side is negative for $r \geq .9$ and thus the inequality holds for $r$ with $.9 \leq r \leq 1$. For $r$ with $0 \leq r \leq .9$ we square both sides.) Thus (18) is smaller than or equal to

$$
\begin{gathered}
\left(\begin{array}{c}
N \\
n
\end{array}\right) \frac{\left(\operatorname{vol}_{n-2}\left(\partial B_{2}^{n-1}\right)\right)^{n-1}}{\left(\operatorname{vol}_{n-1}\left(\partial B_{2}^{n}\right)\right)^{n-2}} \frac{n}{(n-1)^{n-1}} \\
\times \int_{s(1-c)}^{1}(1-s)^{N-n}\left(\frac{1}{2} r^{2}+r^{4}-c\right) r^{(n-1)^{2}} d s .
\end{gathered}
$$

Now we evaluate the integral. We use Lemma 8. By differentiation we verify that $\left(\frac{1}{2} r^{2}+r^{4}-c\right) r^{(n-1)^{2}}$ is a monotone function of $r$. Here we use 
the fact that $\frac{1}{2} r^{2}+r^{4}-c$ is nonnegative. Hence

$$
\begin{aligned}
\int_{s(1-c)}^{1}(1-s)^{N-n}\left(\frac{1}{2} r^{2}\right. & \left.+r^{4}-c\right) r^{(n-1)^{2}} d s \\
\leq & \frac{1}{2} \int_{0}^{1}(1-s)^{N-n}\left(s \frac{\operatorname{vol}_{n-1}\left(\partial B_{2}^{n}\right)}{\operatorname{vol}_{n-1}\left(B_{2}^{n-1}\right)}\right)^{n-1+\frac{2}{n-1}} d s \\
& +\int_{0}^{1}(1-s)^{N-n}\left(s \frac{\operatorname{vol}_{n-1}\left(\partial B_{2}^{n}\right)}{\operatorname{vol}_{n-1}\left(B_{2}^{n-1}\right)}\right)^{n-1+\frac{4}{n-1}} d s \\
& -\int_{0}^{1}(1-s)^{N-n} c\left(s \frac{\operatorname{vol}_{n-1}\left(\partial B_{2}^{n}\right)}{\operatorname{vol}_{n-1}\left(B_{2}^{n-1}\right)}\right)^{n-1} d s \\
& +\int_{0}^{s(1-c)}(1-s)^{N-n} c\left(s \frac{\operatorname{vol}_{n-1}\left(\partial B_{2}^{n}\right)}{\operatorname{vol}_{n-1}\left(B_{2}^{n-1}\right)}\right)^{n-1} d s .
\end{aligned}
$$

By (13),

$$
\begin{aligned}
& \int_{s(1-c)}^{1}(1-s)^{N-n}\left(\frac{1}{2} r^{2}+r^{4}-c\right) r^{(n-1)^{2}} d s \\
& \leq \frac{1}{2}\left(\frac{\operatorname{vol}_{n-1}\left(\partial B_{2}^{n}\right)}{\operatorname{vol}_{n-1}\left(B_{2}^{n-1}\right)}\right)^{n-1+\frac{2}{n-1}} \frac{\Gamma(N-n+1) \Gamma\left(n+\frac{2}{n-1}\right)}{\Gamma\left(N+1+\frac{2}{n-1}\right)} \\
& +\left(\frac{\operatorname{vol}_{n-1}\left(\partial B_{2}^{n}\right)}{\operatorname{vol}_{n-1}\left(B_{2}^{n-1}\right)}\right)^{n-1+\frac{4}{n-1}} \frac{\Gamma(N-n+1) \Gamma\left(n+\frac{4}{n-1}\right)}{\Gamma\left(N+1+\frac{4}{n-1}\right)} \\
& -\left(1-\frac{1}{n^{2}}\right)\left(\frac{\operatorname{vol}_{n-1}\left(\partial B_{2}^{n}\right)}{\operatorname{vol}_{n-1}\left(B_{2}^{n-1}\right)}\right)^{n-1} \frac{\Gamma(N-n+1) \Gamma(n)}{\Gamma(N+1)} \\
& \times \frac{(n-1)^{\frac{n+1}{n-1}}\left(\operatorname{vol}_{n-1}\left(\partial B_{2}^{n}\right)\right)^{\frac{2}{n-1}}}{\left(\operatorname{vol}_{n-2}\left(\partial B_{2}^{n-1}\right)\right)^{\frac{2}{n-1}}} \frac{\Gamma\left(n+1+\frac{2}{n-1}\right)}{2(n+1) !} N^{-\frac{2}{n-1}} \\
& +c s(1-c)\left(\frac{\operatorname{vol}_{n-1}\left(\partial B_{2}^{n}\right)}{\operatorname{vol}_{n-1}\left(B_{2}^{n-1}\right)}\right)^{n-1} \max _{s \in[0, s(1-c)]}(1-s)^{N-n} s^{n-1} .
\end{aligned}
$$

Thus

$$
\begin{aligned}
\int_{s(1-c)}^{1}(1- & s)^{N-n}\left(\frac{1}{2} r^{2}+r^{4}-c\right) r^{(n-1)^{2}} d s \\
& \leq \frac{1}{2}\left(\frac{\operatorname{vol}_{n-1}\left(\partial B_{2}^{n}\right)}{\operatorname{vol}_{n-1}\left(B_{2}^{n-1}\right)}\right)^{n-1+\frac{2}{n-1}} \frac{\Gamma(N-n+1) \Gamma\left(n+\frac{2}{n-1}\right)}{\Gamma\left(N+1+\frac{2}{n-1}\right)}
\end{aligned}
$$




$$
\begin{aligned}
& +\left(\frac{\operatorname{vol}_{n-1}\left(\partial B_{2}^{n}\right)}{\operatorname{vol}_{n-1}\left(B_{2}^{n-1}\right)}\right)^{n-1+\frac{4}{n-1}} \frac{\Gamma(N-n+1) \Gamma\left(n+\frac{4}{n-1}\right)}{\Gamma\left(N+1+\frac{4}{n-1}\right)} \\
& -\frac{1}{2}\left(1-\frac{1}{n^{2}}\right) \frac{n-1}{(n+1) n}\left(\frac{\operatorname{vol}_{n-1}\left(\partial B_{2}^{n}\right)}{\operatorname{vol}_{n-1}\left(B_{2}^{n-1}\right)}\right)^{n-1+\frac{2}{n-1}} \\
& \times \frac{\Gamma(N-n+1) \Gamma\left(n+1+\frac{2}{n-1}\right)}{\Gamma(N+1)} N^{-\frac{2}{n-1}} \\
& +c s(1-c)\left(\frac{\operatorname{vol}_{n-1}\left(\partial B_{2}^{n}\right)}{\operatorname{vol}_{n-1}\left(B_{2}^{n-1}\right)}\right)^{n-1} \max _{s \in[0, s(1-c)]}(1-s)^{N-n} s^{n-1} .
\end{aligned}
$$

The second summand is asymptotically equal to

$$
\begin{aligned}
\left(\frac{\operatorname{vol}_{n-1}\left(\partial B_{2}^{n}\right)}{\operatorname{vol}_{n-1}\left(B_{2}^{n-1}\right)}\right)^{n-1+\frac{4}{n-1}} \frac{(N-n) !(n-1) ! n^{\frac{4}{n-1}}}{N !(N+1)^{\frac{4}{n-1}}} \\
=\left(\frac{\operatorname{vol}_{n-1}\left(\partial B_{2}^{n}\right)}{\operatorname{vol}_{n-1}\left(B_{2}^{n-1}\right)}\right)^{n-1+\frac{4}{n-1}} \frac{n^{-1+\frac{4}{n-1}}}{\left(\begin{array}{c}
N \\
n
\end{array}\right)(N+1)^{\frac{4}{n-1}}} .
\end{aligned}
$$

This summand is of the order $N^{-\frac{4}{n-1}}$ while the others are of the order $N^{-\frac{2}{n-1}}$.

We consider the sum of the first and third summands:

$$
\begin{aligned}
& \frac{1}{2}\left(\frac{\operatorname{vol}_{n-1}\left(\partial B_{2}^{n}\right)}{\operatorname{vol}_{n-1}\left(B_{2}^{n-1}\right)}\right)^{n-1+\frac{2}{n-1}} \frac{\Gamma(N-n+1) \Gamma\left(n+\frac{2}{n-1}\right)}{\Gamma\left(N+1+\frac{2}{n-1}\right)} \\
& \quad \times\left(1-\left(1-\frac{1}{n^{2}}\right) \frac{(n-1) \Gamma\left(n+1+\frac{2}{n-1}\right) \Gamma\left(N+1+\frac{2}{n-1}\right)}{n(n+1) \Gamma\left(n+\frac{2}{n-1}\right) \Gamma(N+1) N^{\frac{2}{n-1}}}\right)
\end{aligned}
$$

Since $\Gamma\left(n+1+\frac{2}{n-1}\right)=\left(n+\frac{2}{n-1}\right) \Gamma\left(n+\frac{2}{n-1}\right)$ the latter expression equals

$$
\begin{aligned}
& \frac{1}{2}\left(\frac{\operatorname{vol}_{n-1}\left(\partial B_{2}^{n}\right)}{\operatorname{vol}_{n-1}\left(B_{2}^{n-1}\right)}\right)^{n-1+\frac{2}{n-1}} \frac{\Gamma(N-n+1) \Gamma\left(n+\frac{2}{n-1}\right)}{\Gamma\left(N+1+\frac{2}{n-1}\right)} \\
& \quad \times\left(1-\left(1-\frac{1}{n^{2}}\right) \frac{(n-1)\left(n+\frac{2}{n-1}\right) \Gamma\left(N+1+\frac{2}{n-1}\right)}{n(n+1) \Gamma(N+1) N^{\frac{2}{n-1}}}\right) .
\end{aligned}
$$

Since $\Gamma\left(N+1+\frac{2}{n-1}\right)$ is asymptotically equal to $(N+1)^{\frac{2}{n-1}} \Gamma(N+1)$ the sum of the first and third summands is for large $N$ of the order

$$
\frac{1}{n}\left(\frac{\operatorname{vol}_{n-1}\left(\partial B_{2}^{n}\right)}{\operatorname{vol}_{n-1}\left(B_{2}^{n-1}\right)}\right)^{n-1+\frac{2}{n-1}} \frac{\Gamma(N-n+1) \Gamma\left(n+\frac{2}{n-1}\right)}{\Gamma\left(N+1+\frac{2}{n-1}\right)}
$$


which in turn is of the order

$$
\frac{1}{n^{2}}\left(\frac{\operatorname{vol}_{n-1}\left(\partial B_{2}^{n}\right)}{\operatorname{vol}_{n-1}\left(B_{2}^{n-1}\right)}\right)^{n-1+\frac{2}{n-1}}\left(\begin{array}{c}
N \\
n
\end{array}\right)^{-1} N^{-\frac{2}{n-1}} .
$$

We now consider the fourth summand. By (14) and (20) it is less than

$$
b N^{-\frac{2}{n-1}} \frac{n}{e^{5 / 6} N}\left(\frac{\operatorname{vol}_{n-1}\left(\partial B_{2}^{n}\right)}{\operatorname{vol}_{n-1}\left(B_{2}^{n-1}\right)}\right)^{n-1} \max _{s \in[0, s(1-c)]}(1-s)^{N-n} s^{n-1} .
$$

The maximum of the function $(1-s)^{N-n} s^{n-1}$ is attained at $(n-1) /(N-1)$ and the function is increasing on the interval $[0,(n-1) /(N-1)]$. Therefore, by (20) we have $s(1-c)<(n-1) /(N-1)$ and the maximum of this function over the interval $[0, s(1-c)]$ is attained at $s(1-c)$. By $(20)$ we have $s(1-c) \leq e^{1 / 6} \frac{n}{e N}$ and thus for $N$ sufficiently large

$$
\begin{aligned}
\max _{s \in[0, s(1-c)]}(1-s)^{N-n} s^{n-1} & \leq\left(1-\frac{n}{e^{5 / 6} N}\right)^{N-n}\left(e^{1 / 6} \frac{n}{e N}\right)^{n-1} \\
& \leq \exp \left(\frac{n-1}{6}-\frac{n(N-n)}{e^{5 / 6} N}\right)\left(\frac{n}{e N}\right)^{n-1} \\
& \leq \exp \left(-\frac{n}{6}\right)\left(\frac{n}{e N}\right)^{n-1} .
\end{aligned}
$$

Thus we get for (26) the bound, with a new constant $b$,

$$
b N^{-\frac{2}{n-1}}\left(\frac{\operatorname{vol}_{n-1}\left(\partial B_{2}^{n}\right)}{\operatorname{vol}_{n-1}\left(B_{2}^{n-1}\right)}\right)^{n-1} e^{-n / 6} \frac{n^{n} e^{-n}}{N^{n}} .
$$

This is asymptotically equal to

$$
b N^{-\frac{2}{n-1}}\left(\frac{\operatorname{vol}_{n-1}\left(\partial B_{2}^{n}\right)}{\operatorname{vol}_{n-1}\left(B_{2}^{n-1}\right)}\right)^{n-1} e^{-n / 6} \frac{1}{\left(\begin{array}{l}
N \\
n
\end{array}\right) \sqrt{2 \pi n}} .
$$

Altogether, (15) for $N$ sufficiently large can be estimated by

$$
\begin{aligned}
\left(\begin{array}{l}
N \\
n
\end{array}\right) \frac{\left(\operatorname{vol}_{n-2}\left(\partial B_{2}^{n-1}\right)\right)^{n-1}}{\left(\operatorname{vol}_{n-1}\left(\partial B_{2}^{n}\right)\right)^{n-2}} \frac{n}{(n-1)^{n-1}} \\
\times\left\{\frac{1}{n^{2}}\left(\frac{\operatorname{vol}_{n-1}\left(\partial B_{2}^{n}\right)}{\operatorname{vol}_{n-1}\left(B_{2}^{n-1}\right)}\right)^{n-1+\frac{2}{n-1}}\left(\begin{array}{c}
N \\
n
\end{array}\right)^{-1} N^{-\frac{2}{n-1}}\right. \\
\left.+b N^{-\frac{2}{n-1}}\left(\frac{\operatorname{vol}_{n-1}\left(\partial B_{2}^{n}\right)}{\operatorname{vol}_{n-1}\left(B_{2}^{n-1}\right)}\right)^{n-1} e^{-n / 6} \frac{1}{\left(\begin{array}{l}
N \\
n
\end{array}\right) \sqrt{2 \pi n}}\right\}
\end{aligned}
$$

This can be estimated by a constant times

$$
\left(\operatorname{vol}_{n-1}\left(\partial B_{2}^{n}\right)\right) n\left\{\frac{1}{n^{2}} N^{-\frac{2}{n-1}}+b N^{-\frac{2}{n-1}} e^{-n / 6} \frac{1}{\sqrt{2 \pi n}}\right\} .
$$


Finally, it should be noted that we have been estimating the approximation of $B_{2}^{n}(1-c)$ and not that of $B_{2}^{n}$. Therefore, we need to multiply (28) by $(1-c)^{-n}$. By $(14)$

$$
(1-c)^{n} \geq 1-b \frac{n}{N^{\frac{2}{n-1}}}
$$

so that for all $N$ with $N \geq(2 b n)^{\frac{n-1}{2}}$ we have $(1-c)^{-n} \leq 2$.

3. Proof of Theorem 2. We need another lemma.

Lemma 9. Let $P_{M}$ be a polytope with $M$ facets $F_{1}, \ldots, F_{M}$ that is best approximating for a convex body $K$ in $\mathbb{R}^{n}$ with respect to the symmetric difference metric. For $k=1, \ldots, M$, let

$$
F_{k}^{\mathrm{i}}=F_{k} \cap K, \quad F_{k}^{\mathrm{a}}=F_{k} \cap K^{\mathrm{c}} .
$$

Then, for all $j=1, \ldots, M$,

$$
\operatorname{vol}_{n-1}\left(F_{j}^{\mathrm{i}}\right)=\operatorname{vol}_{n-1}\left(F_{j}^{\mathrm{a}}\right) .
$$

Proof. Let $H_{j}, j=1, \ldots, M$, be the hyperplane containing the face $F_{j}$. Then

$$
P_{M}=\bigcap_{j=1}^{M} H_{j}^{+} .
$$

Suppose $H_{k}=H\left(x_{k}, \xi_{k}\right)$, i.e. $H_{k}$ is the hyperplane containing $x_{k}$ and orthogonal to $\xi_{k}$. We consider

$$
P_{t}=\bigcap_{j \neq k} H_{j}^{+} \cap H^{+}\left(x_{k}+\frac{t}{\left\|x_{k}\right\|} x_{k}, \xi_{k}\right) .
$$

We have

$$
\operatorname{vol}_{n-1}\left(P_{t} \triangle K\right)=\operatorname{vol}_{n-1}\left(P_{M} \triangle K\right)+t\left(\operatorname{vol}_{n-1}\left(F_{k}^{\mathrm{a}}\right)-\operatorname{vol}_{n-1}\left(F_{k}^{\mathrm{i}}\right)\right)+\psi(t)
$$

where $\psi(t) / t^{2}$ is a bounded function.

Proof of Theorem 2. Let $P_{M}$ be a best approximating polytope with $M$ facets $F_{1}, \ldots, F_{M}$ for $B_{2}^{n}$ with respect to the symmetric difference metric. For $k=1, \ldots, M$, let

$$
F_{k}^{\mathrm{i}}=F_{k} \cap B_{2}^{n}, \quad F_{k}^{\mathrm{a}}=F_{k} \cap\left(B_{2}^{n}\right)^{\mathrm{c}},
$$

let $H_{k}$ be the hyperplane containing the facet $F_{k}$ and let $C_{k}$ be the cap of $B_{2}^{n}$ with base $H_{k} \cap B_{2}^{n}$. (There are actually two caps, we take the one whose interior has empty intersection with $P_{M}$.) For $k=1, \ldots, M$ we put

$$
h_{k}= \begin{cases}\text { height of the cap } C_{k} & \text { if } F_{k} \cap\left(B_{2}^{n}\right)^{\circ} \neq \emptyset, \\ 0, & \text { if } F_{k} \cap\left(B_{2}^{n}\right)^{\circ}=\emptyset .\end{cases}
$$


Then

$$
\operatorname{vol}_{n-1}\left(P_{M} \triangle B_{2}^{n}\right) \geq \frac{1}{n} \sum_{k=1}^{M} h_{k} \operatorname{vol}_{n-1}\left(F_{k}^{\mathrm{i}}\right) .
$$

Let $r_{k}$ be such that $\operatorname{vol}_{n-1}\left(r_{k} B_{2}^{n-1}\right)=\operatorname{vol}_{n-1}\left(F_{k}^{\mathrm{i}}\right)$. Thus

$$
r_{k}=\left(\frac{\operatorname{vol}_{n-1}\left(F_{k}^{\mathrm{i}}\right)}{\operatorname{vol}_{n-1}\left(B_{2}^{n-1}\right)}\right)^{\frac{1}{n-1}}
$$

Let $\widetilde{h}_{k}$ be the height of the cap of $B_{2}^{n}$ with base $r_{k} B_{2}^{n-1}$. Then

$$
\widetilde{h}_{k} \leq h_{k} \quad \text { for all } k \text {, }
$$

and

$$
\widetilde{h}_{k} \geq \frac{1}{2} r_{k}^{2} \geq \frac{1}{2}\left(\frac{\operatorname{vol}_{n-1}\left(F_{k}^{\mathrm{i}}\right)}{\operatorname{vol}_{n-1}\left(B_{2}^{n-1}\right)}\right)^{\frac{2}{n-1}} .
$$

Thus from (29) with (30) we get

$$
\begin{aligned}
\operatorname{vol}_{n-1}\left(P_{M} \triangle B_{2}^{n}\right) & \geq \frac{1}{2 n} \sum_{k=1}^{M} \frac{\left(\operatorname{vol}_{n-1}\left(F_{k}^{\mathrm{i}}\right)\right)^{\frac{n+1}{n-1}}}{\left(\operatorname{vol}_{n-1}\left(B_{2}^{n-1}\right)\right)^{\frac{2}{n-1}}} \\
& \geq \frac{1}{8 \pi e} \sum_{k=1}^{M}\left(\operatorname{vol}_{n-1}\left(F_{k}^{\mathrm{i}}\right)\right)^{\frac{n+1}{n-1}}
\end{aligned}
$$

We consider two cases. The first case is

$$
\sum_{k=1}^{M} \operatorname{vol}_{n-1}\left(F_{k}^{\mathrm{i}}\right)+\sum_{k=1}^{M} \operatorname{vol}_{n-1}\left(F_{k}^{\mathrm{a}}\right) \geq c \operatorname{vol}_{n-1}\left(\partial B_{2}^{n}\right)
$$

where $M \geq 10^{(n-1) / 2}$ and $c=9 / 10$. It then follows from Lemma 9 that

$$
\sum_{k=1}^{M} \operatorname{vol}_{n-1}\left(F_{k}^{\mathrm{i}}\right) \geq \frac{c}{2} \operatorname{vol}_{n-1}\left(\partial B_{2}^{n}\right) .
$$

By Hölder's inequality

$$
\sum_{k=1}^{M} \operatorname{vol}_{n-1}\left(F_{k}^{\mathrm{i}}\right) \leq\left(\sum_{k=1}^{M}\left(\operatorname{vol}_{n-1}\left(F_{k}^{\mathrm{i}}\right)\right)^{p}\right)^{1 / p} M^{1 / p^{\prime}}
$$

Therefore from (31) and (33) with $p=\frac{n+1}{n-1}$ we get

$$
\operatorname{vol}_{n-1}\left(P_{M} \triangle B_{2}^{n}\right) \geq \frac{(c / 2)^{\frac{n+1}{n-1}}}{8 \pi e} \frac{1}{M^{\frac{2}{n-1}}}\left(n \operatorname{vol}_{n}\left(B_{2}^{n}\right)\right)^{\frac{n+1}{n-1}} \geq \frac{c^{\frac{n+1}{n-1}}}{8 M^{\frac{2}{n-1}}} \operatorname{vol}_{n}\left(B_{2}^{n}\right)
$$


The second case is that (32) does not hold. Thus

$$
\sum_{k=1}^{M} \operatorname{vol}_{n-1}\left(F_{k}\right)=\sum_{k=1}^{M} \operatorname{vol}_{n-1}\left(F_{k}^{\mathrm{i}}\right)+\sum_{k=1}^{M} \operatorname{vol}_{n-1}\left(F_{k}^{\mathrm{a}}\right)<c \operatorname{vol}_{n-1}\left(\partial B_{2}^{n}\right) .
$$

Then, by the isoperimetric inequality,

$$
\operatorname{vol}_{n}\left(P_{M}\right) \leq\left(\frac{\sum_{k=1}^{M} \operatorname{vol}_{n-1}\left(F_{k}\right)}{\operatorname{vol}_{n-1}\left(\partial B_{2}^{n}\right)}\right)^{\frac{n}{n-1}} \operatorname{vol}_{n}\left(B_{2}^{n}\right)<c^{\frac{n}{n-1}} \operatorname{vol}_{n}\left(B_{2}^{n}\right)
$$

and thus

$$
\operatorname{vol}_{n}\left(P_{M} \triangle B_{2}^{n}\right) \geq\left(1-c^{\frac{n}{n-1}}\right) \operatorname{vol}_{n}\left(B_{2}^{n}\right) .
$$

Since $c=9 / 10$, this last expression is greater than $M^{-\frac{2}{n-1}} \operatorname{vol}_{n}\left(B_{2}^{n}\right)$, provided $M \geq 10^{\frac{n-1}{2}}$, which holds by assumption.

\section{References}

[Bö] K. Böröczky, Polytopal approximation bounding the number of $k$-faces, J. Approx. Theory 102 (2000), 263-285.

[GRS1] Y. Gordon, S. Reisner and C. Schütt, Umbrellas and polytopal approximation of the Euclidean ball, ibid. 90 (1997), 9-22.

[GRS2] - - - —, Erratum to [GRS1], ibid. 95 (1998), 331.

[Gr1] P. M. Gruber, Approximation of convex bodies, in: P. M. Gruber and J. M. Wills (eds.), Convexity and its Applications, Birkhäuser, 1983, 131-162.

[Gr2] - , Asymptotic estimates for best and stepwise approximation of convex bodies II, Forum Math. 5 (1993), 521-538.

[Gr3] -, Aspects of approximation of convex bodies, in: P. M. Gruber and J. M. Wills (eds.), Handbook of Convex Geometry, North-Holland, 1993, 319-345.

[Lud] M. Ludwig, Asymptotic approximation of smooth convex bodies by general polytopes, Mathematika 46 (1999), 103-125.

[MaS1] P. Mankiewicz and C. Schütt, A simple proof of an estimate for the approximation of the Euclidean ball and the Delone triangulation numbers, J. Approx. Theory 107 (2000), 268-280.

[MaS2] -, -, On the Delone triangulation numbers, ibid. 111 (2001), 139-142.

$[\mathrm{McV}]$ D. E. McClure and R. Vitale, Polygonal approximation of plane convex bodies, J. Math. Anal. Appl. 51 (1975), 326-358.

[Mil] R. E. Miles, Isotropic random simplices, Adv. Appl. Probab. 3 (1971), 353-382.

[Mü] J. S. Müller, Approximation of the ball by random polytopes, J. Approx. Theory 63 (1990), 198-209.

[Sch] C. Schütt, Random polytopes and affine surface area, Math. Nachr. 170 (1994), 227-249.

[SW] C. Schütt and E. Werner, Polytopes with vertices chosen randomly from the boundary of a convex body, in: Israel Seminar 2001-2002, V. D. Milman and G. Schechtman (eds.), Lecture Notes in Math. 1807, Springer, 2003, 241-422.

[Wen] J. G. Wendel, A problem in geometric probability, Math. Scand. 11 (1962), 109-111. 
[Zä] M. Zähle, A kinematic formula and moment measures of random sets, Math. Nachr. 149 (1962), 325-340.

Institut für Diskrete Mathematik und Geometrie Technische Universität Wien

Wiedner Hauptstraße 8-10/104

1040 Wien, Austria

E-mail: monika.ludwig@tuwien.ac.at

Mathematisches Seminar

Christian Albrechts Universität

D-24098 Kiel, Germany

E-mail: schuett@math.uni-kiel.de
Department of Mathematics Case Western Reserve University Cleveland, OH 44106, U.S.A. E-mail: emw2@po.cwru.edu and

Université de Lille 1 UFR de Mathématique 59655 Villeneuve d'Ascq, France

Received August 9, 2004

Revised version November 3, 2005 